\title{
Electrode and Langmuir probe tools used for flow damping studies in the Helically Symmetric Experiment
}

\author{
S. P. Gerhardt, ${ }^{\text {a) }}$ D. T. Anderson, F. S. B. Anderson, and J. N. Talmadge \\ Department of Electrical and Computer Engineering, University of Wisconsin, Madison, Wisconsin 53703
}

(Received 9 May 2004; accepted 25 July 2004; published 1 November 2004)

\begin{abstract}
A system of electrodes and Langmuir probes has been developed for the measurement of plasma flow damping in the Helically Symmetric Experiment (HSX) stellarator. A biased electrode is used to apply a $\mathbf{J} \times \mathbf{B}$ torque to the plasma. The fast switching electrode power supply allows the electrode voltage to be applied in $\sim 1 \mu \mathrm{s}$, which is much faster than any of the plasma time scales; the electrode current is terminated on a similar time scale at the end of the electrode pulse. A pair of multitipped Mach probes have been designed to measure the plasma flows in a magnetic surface with good spatial and time resolution $(\Delta t>20 \mu \mathrm{s})$. The unmagnetized model by Hutchinson is used to analyze the Mach probe data, and radial force balance is found to be well satisfied during electrode bias. These probes allow for measurements of the transient response of the plasma flows and floating potential when the electrode is energized. An example of using the system for the estimation of viscous damping times in HSX is provided. (C) 2004 American Institute of Physics. [DOI: 10.1063/1.1804832]
\end{abstract}

\section{INTRODUCTION}

Measurements of plasma flow damping are a critical component of the experimental program of the Helically Symmetric Experiment (HSX). ${ }^{1}$ HSX is the world's first stellarator ${ }^{2}$ with a direction of symmetry; ${ }^{3}$ there is minimal variation of $|B|$ along a helical path on a magnetic surface, defining the direction of symmetry. The neoclassical viscous damping of flows along this direction is predicted to be very low. This is in contrast to a conventional stellarator, where the complicated variation of $|B|$ leads to large viscous damping of plasma flows in all directions on a magnetic surface. ${ }^{4}$ Given the importance of plasma flows and electric field shear in the suppression of turbulence, ${ }^{5}$ a detailed study of flow damping in HSX is a topic of great importance. This article describes the system of tools used to manipulate and study these plasma flows.

The plasma flows are generated using an electrode inserted inside the last closed magnetic surface and biased with respect to the vacuum vessel. Currents drawn by the electrode flow down the shaft of the electrode, through the power supply, to the vacuum vessel, and back through the plasma to the electrode. This "return current" flowing through the plasma applies a torque to the plasma, leading to plasma rotation. This biasing technique was pioneered on the B-3 stellarator ${ }^{6}$ and has been used to great advantage at, for instance, CCT, ${ }^{7}$ TEXTOR, ${ }^{8}$ Phaedrus-T, ${ }^{9}$ TUMAN-3, ${ }^{10}$ T- $10,{ }^{11}$ ISSTOK, ${ }^{12} \mathrm{RFX}_{,}^{13} \mathrm{MST}^{14}{ }^{14} \mathrm{CAT},{ }^{15}$ and IMS. ${ }^{16}$ Of the incomplete sampling of biased electrode experiments given above, it appears that only the systems at IMS and MST were designed to allow the transient phase of the plasma spin-up or spin-down to be studied. As will be shown later, the fast time

\footnotetext{
a)Present address: Princeton Plasma Physics Laboratory, Princeton, NJ
} 08543; electronic mail: sgerhard@pppl.gov scale evolution ( $\Delta t \geqslant 20 \mu \mathrm{s})$ of plasma flows, potentials, and the electrode current allow many features of the plasma dynamics to be investigated. The bias power supply and plasma diagnostic systems at HSX have been designed with these fast time scale measurements in mind.

Langmuir probes and Doppler spectroscopy are the most common means of studying the mass flow in fusion plasmas. In Doppler spectroscopy, ${ }^{17}$ the wavelength shift of a spectral line is used to measure the average velocity of the plasma species which emitted the light. In its most common manifestation, collection optics outside the plasma gather light from along a chord. This light is input to a spectrometer, where it is dispersed and an array of detectors is used to determine the spectral distribution of the light. These systems have a spatial resolution which is often limited by the chordal integration of the plasma light emission and a time resolution limited by the readout time of multielement detectors. The spatial resolution of such systems can be improved using charge exchange spectroscopy, ${ }^{18}$ while the time resolution can be improved by using arrays of photomultiplier tubes as detectors. ${ }^{19}$ Both of these solutions add significant cost and complexity to the system.

Given these difficulties with the spectroscopic measurements, we have designed and constructed a Mach probe system for the measurement of plasma flows. The probes are of the Gundestrup ${ }^{20}$ variety, and are able to measure toroidal and poloidal flow simultaneously. Compared to the complicated spectroscopic systems noted earlier, these probes have the advantage of good spatial and time resolution at minimal cost. Two of these probes have been constructed, enabling the flow parameters to be studied at two locations simultaneously.

The layout of the remainder of this article is as follows. Section II provides details of the plasma biasing system, including the electrode and power supply design. Section III 


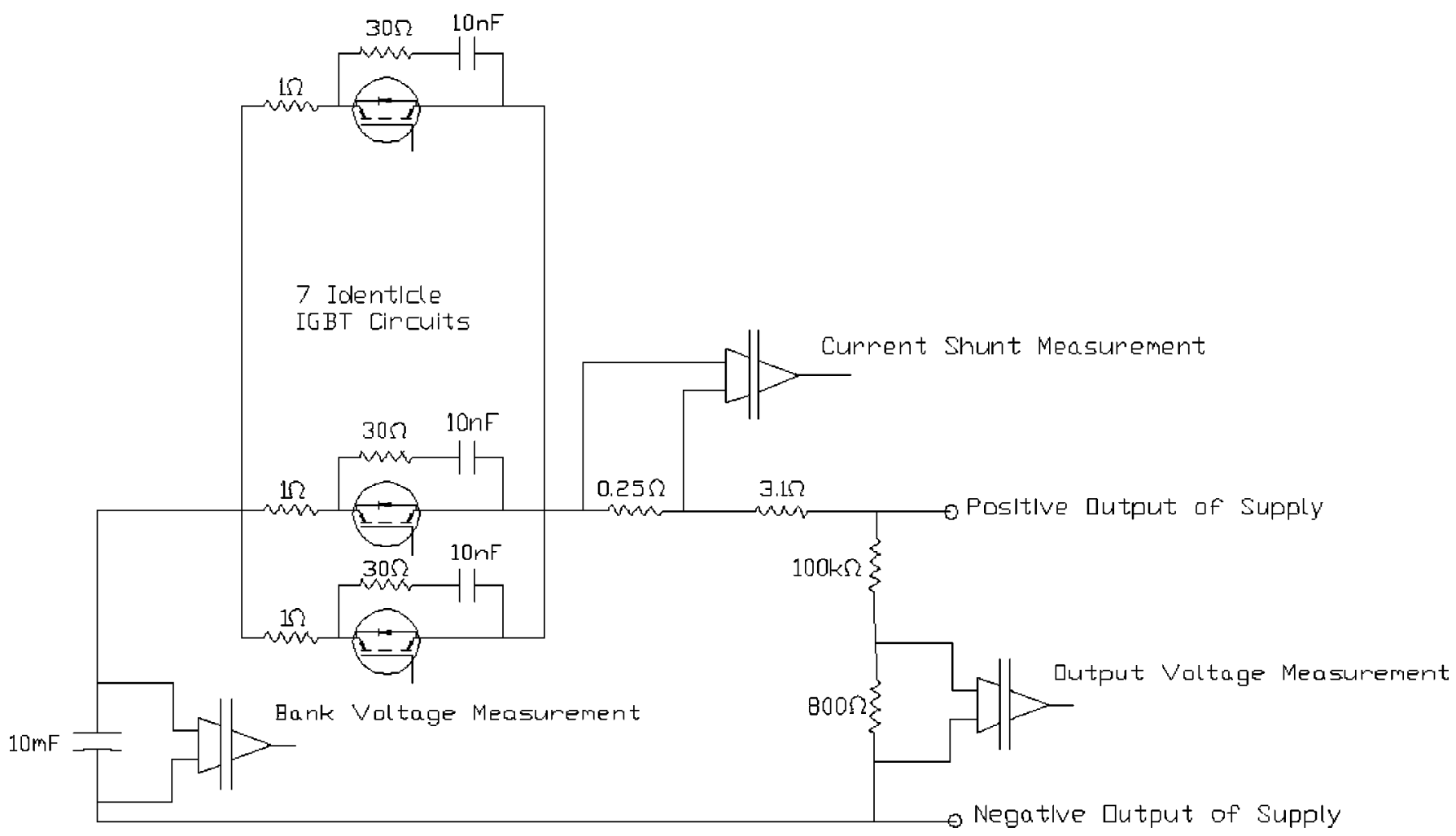

FIG. 1. Fast switching circuit of the power supply. Seven identical IGBT switching circuits are used to switch the power supply on and off in $\sim 1 \mu$ s, while a voltage divider and current shunt are used to measure the electrode voltage and current.

describes the multitipped Mach probes used to measure the plasma flow. Details of both the construction of the probes and the interpretation of the data are provided. Section IV provides an example of the use of the system to study damping of plasma flows in HSX. Throughout this article, we use the following convention to distinguish between probes and electrodes, following Ref. 9. Probes are designed to measure plasma parameters with a minimal perturbation. Electrodes are designed to induce macroscopic changes in the plasma.

$\mathrm{HSX}^{1}$ is a medium size, four field period stellarator experiment at the University of Wisconsin-Madison. HSX has a major radius of $1.2 \mathrm{~m}$ and a minor radius of $\sim 0.10 \mathrm{~m}$, varying slightly with the machine configuration. The magnetic field on axis is $0.5 \mathrm{~T}$ in the experiments described in this article. Plasma is produced using approximately $50 \mathrm{~kW}$ of second harmonic electron cyclotron heating at $28 \mathrm{GHz}$. HSX is operated in the standard quasihelically symmetric configuration and hydrogen is the working gas for all data presented in this article.

\section{THE BIASED ELECTRODE SYSTEM FOR HSX}

To induce plasma flows in HSX, we use a biased electrode system. The electrode is a molybdenum cylinder, 1.9 $\mathrm{cm}$ long and $1.9 \mathrm{~cm}$ in diameter. This cylinder is captured inside a boron nitride shroud, allowing only $0.635 \mathrm{~cm}$ of the length of the electrode at the end of the cylinder to be exposed to the plasma. This electrode assembly is mounted on a $0.635 \mathrm{~cm}$ diameter oxygen-free high-conductivity copper shaft, which is in turn joined to a single pin, medium current feedthrough (MDC Vacuum Products part No. 640001). The shaft and feedthrough are protected from plasma by boron nitride armor. This entire assembly is mounted inside a welded bellows, and can be positioned in the plasma using a linear positioning stage. The electrode typically requires some conditioning before being used in electron cyclotron heated $(\mathrm{ECH})$ plasmas. This is generally accomplished by grounding it to the vessel and then inserting it into the helium glow discharge plasma that conditions the HSX plasma facing surfaces.

The power supply consists of a capacitor bank, control electronics, and diagnostic outputs. The capacitor bank provides $10 \mathrm{mF}$ of capacitance with a maximum voltage of 900 $\mathrm{V}$. To charge the bank, a $600 \mathrm{~V}, 1.6 \mathrm{~A}$ power supply is used, setting the upper limit on what bias voltages can be applied. The bank is charged before each plasma shot, and the voltage remaining on the capacitors is removed via a shorting bar at the end of each discharge.

The fast switching circuit in the power supply is illustrated in Fig. 1. The capacitor bank voltage is switched onto the electrode using seven parallel solid state switches. Each insulated gate bipolar transistor (IGBT) switch (International Rectifier, IRG4PH50UD) is capable of standing off $1200 \mathrm{~V}$ and conducting 45 A direct current. The combination of a 30 $\Omega$ resistor and $10 \mathrm{nF}$ capacitor in parallel with each IGBT is used to snub out voltage ringing when the current is shut off, while $1 \Omega$ resistors in series with the switches cause the current to distribute evenly between the seven IGBT circuits. With these IGBT switches, the bias voltage can be turned on in $\approx 1 \mu$ s at the beginning of the pulse, while the current can be terminated on the same time scale at the end of the pulse. This switching system also allows the bank to be pulsed multiple times during each discharge, increasing the amount 
a)

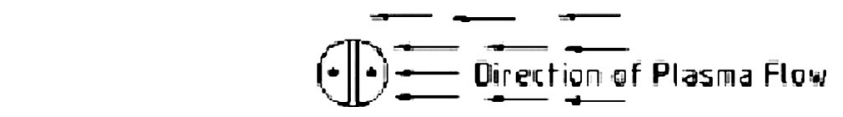

Downstiream Tip - Ceranic Insulator

b)

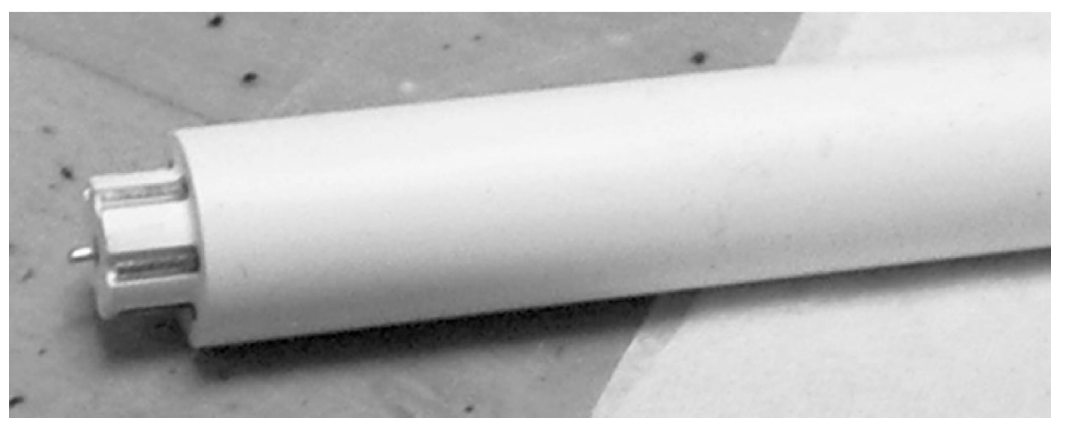

FIG. 2. (a) The design of a simple Mach probe, composed of two Langmuir probe tips biased into ion saturation current and shielded from each other by an insulator and (b) photograph of the six-tipped Mach probe used in HSX. The six tips are arrayed around the body of a boron nitride insulator. A seventh "proud" pin can be used to measure the local floating potential.

of data taken during each plasma discharge. Extra series resistance of $3.35 \Omega$ limits the current to at most $180 \mathrm{~A}$ in the case of an external fault.

In normal operation, the voltage at the electrode, the voltage on the capacitor bank, and the voltage at the output of the power supply are monitored using voltage dividers and Analog Devices AD215BY transformer coupled isolation amplifiers. This system enables these voltages to be monitored with $\approx 120 \mathrm{kHz}$ of bandwidth. The current drawn by the bank is monitored via a Pearson current transformer, enabling very high bandwidth $(>1 \mathrm{MHz})$ measurements of the electrode current for short pulses of the power supply. A 0.25 $\Omega$ current shunt is also installed in the supply. This measurement is limited by both the inductance of the wire wound resistor and the comparatively low bandwidth of the necessary isolation amplifiers (also AD215BY with $120 \mathrm{kHz}$ of bandwidth). Hence, the current shunt is a less attractive measurement than the current transformer, and is generally not used. Examples of the measurements made with these diagnostic systems are provided in Sec. IV.

\section{MULTISIDED MACH PROBES USED IN HSX}

In its simplest manifestation, a Mach probe ${ }^{21}$ is composed of two probe tips separated from each other by an insulator, each biased into ion saturation current $\left(I_{\text {sat }}\right)$. An example of this type of probe is shown in Fig. 2(a). If the plasma is not flowing, then the two tips should collect the same $I_{\text {sat }}$. If the plasma is flowing across the probe as indicated in the figure, then the tip facing the flow $\left(I_{\text {up }}\right)$ will draw more current then the tip facing away from the flow $\left(I_{\text {down }}\right)$. The relationship between the current drawn by the tips and the plasma flow speed is known as the Mach probe calibration problem. In general, the models which relate the upstream and downstream currents to flow speed reduce to an expression of the form

$$
\frac{I_{\text {up }}}{I_{\text {down }}}=\exp (k M) \text {. }
$$

Here, $M$ is the Mach number of the flow $\left[M=V_{\text {flow }} / c_{s}, c_{s}\right.$ $\left.=\operatorname{sqrt}\left(2 T_{e} / m_{i}\right)\right]$ and $k$ is a constant determined by the model used.

This simple Mach probe is only sensitive to flow along the line made by connecting the two tips. Hence, it is necessary to rotate it on a shot to shot basis to measure the total flow vector. This problem has been addressed by the construction of multitipped Mach probes. These probes are composed of many outward facing tips shadowed from each other by the body of the probe, and are known as "Gundestrup probes." $20,22,23$ This style of probe has been selected for making flow measurements in HSX.

We have constructed two "Gundestrup" style probes for HSX. A photograph of one of these probes is shown in Fig. 2(b). Six tips are mounted in a circle facing outward from the insulating boron nitride body of the probe. A seventh tip is a "proud" tip extending beyond the insulating body. This tip is sensitive to the plasma in all directions and is generally used to measure the floating potential $\left(V_{f}\right)$. The tips of the probes are composed of $0.75 \mathrm{~mm}$ diameter tungsten wire; the six tips on the edge have been ground down so that a flat surface faces the plasma. The length of the outward facing tips is typically $0.51 \mathrm{~cm}$ with the proud tip extending typically $0.254 \mathrm{~cm}$ beyond the end of the boron nitride probe head. The diameter of the boron nitride probe head is $0.95 \mathrm{~cm}$ and $1.27 \mathrm{~cm}$ for the two different probes, and is $8.9 \mathrm{~cm}$ long. In each case, the probe head is mounted in a stainless steel tube which is attached to a rotary motion feedthrough (MDC part No. \# BRM-133). This allows the probe to be rotated $\pm 90^{\circ}$. The entire assembly is placed inside a welded bellows and mounted on a sliding assembly to allow precise insertion into the HSX plasma. The signals are carried inside the vacuum using kapton coated wire, and brought out of the machine using a 15 pin $D$-connector feedthrough. 
The six radial tips are biased with respect to the HSX vessel at $-180 \mathrm{~V}$, so that ion saturation current $\left(I_{\mathrm{sat}}\right)$ is collected. Swept probe measurements have shown that this bias voltage is sufficient to collect $I_{\text {sat }}$. Special amplifiers have been constructed for signal isolation and conditioning. Isolation is provided by Analog Devices AD215BY isolation amplifiers as in the power supply. This is followed by a four pole low pass filter with a cutoff at $100 \mathrm{kHz}$. All six Mach probe signals are recorded on the same digitizer, typically at $200 \mathrm{kHz}$, while the floating potential signals from the proud pin are recorded separately at typically $600 \mathrm{kHz}$.

The two Mach probes constructed for HSX are inserted into very different locations in the machine. Because of the helical structure of $|B|$ in HSX, both the low and high magnetic field regions of the device appear on the outboard side at different toroidal angles. One Mach probe is inserted from the outboard side into a region of low $|B|$. The second Mach probe is inserted from the outboard side at a toroidal angle $\sim 135^{\circ}$ separated from the first probe. At this location, the high $|B|$ region corresponds to the outboard side. We are thus able to simultaneously measure flows in the high and low $|B|$ regions of the torus.

As noted at the beginning of this section, a model is required in order to extract flow speeds and directions from the $I_{\text {sat }}$ measurements. As a first step, it is necessary to consider the extent to which ion collection is impacted by the presence of the magnetic field. ${ }^{21}$ If the ion gyroradius $\left(\rho_{i}\right)$, is much greater than the characteristic dimension of the probe $\left(r_{p}\right)$, ions are considered to be unmagnetized. If the opposite limit holds, the ions are considered to be magnetized. For the highly magnetized case $\left(\rho_{i} / r_{p} \ll 1\right)$, the magnetized model by Hutchinson ${ }^{24}$ is the most widely used. In the unmagnetized case, the one dimensional model of Hudis and Lidsky ${ }^{25}$ has been the most widely used in the past. Recent work by Hutchinson has criticized ${ }^{26}$ the Hudis and Lidsky model for its one dimensional nature, and provided a two-dimensional model $^{27}$ for the interpretation of data. Other expressions for the Mach probe calibration can be found in works by Shats et $a l .{ }^{28}$ or Hsu et $a .^{29}$ for the unmagnetized case.

The article by Peterson ${ }^{21}$ provides tests to determine the magnetization. Applying these tests to the Mach probe data from HSX has enabled the determination that the ion collection can be regarded as unmagnetized.

The Mach probe data in this work is analyzed using the unmagnetized model proposed by Hutchinson. ${ }^{27}$ In this model, the ion flux to a negatively biased sphere in a flowing plasma is calculated as a function of angle, using a specialized particle in cell (PIC) code. The calculations are repeated for different values of $T_{i} / T_{e}$ and ion flow speed, $M$ $=V /\left(Z T_{e} / m_{i}\right)^{1 / 2}$, where $V$ is the flow speed in $\mathrm{m} / \mathrm{s}$. There is an assumption of vanishingly small Debye length $\left(\lambda_{D} / r_{p}\right.$ $\ll 1)$. This assumption is well satisfied for HSX, where $\lambda_{D} / r_{p}=\sim 0.01 \ll 1$ As described in the original article, ${ }^{27}$ the PIC code reproduces previous analytical results for a spherical probe in a finite $T_{i}$, stationary plasma. Based on the results of the code in a flowing plasma, a reasonable fit for the ion flux to the sphere in a flowing plasma as a function of angle and flow speed is given by the expression

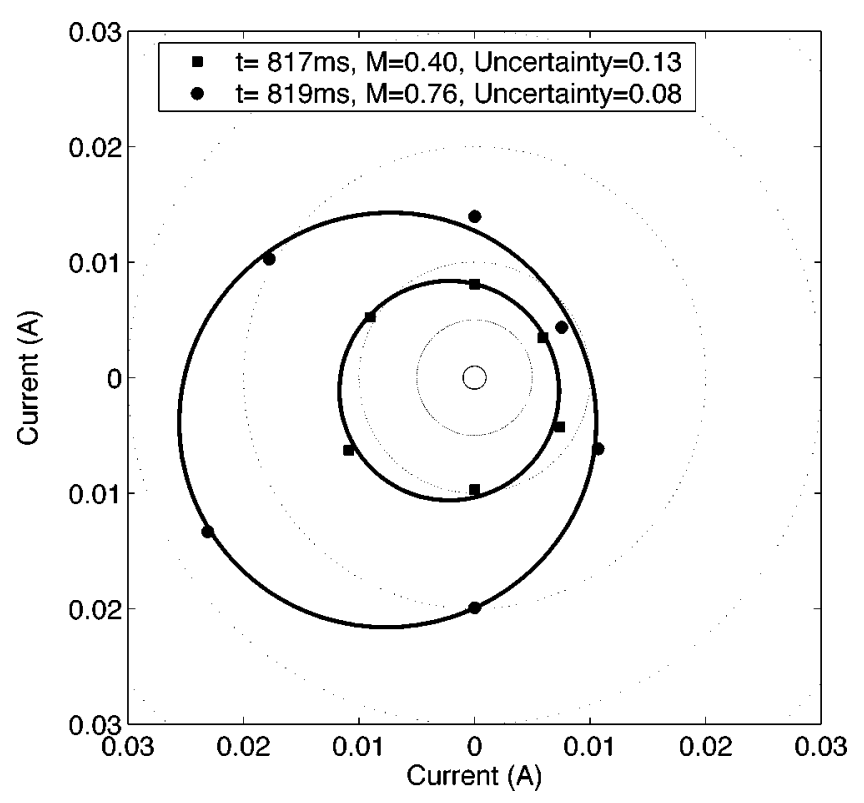

FIG. 3. Examples of the Hutchinson fit at two different time slices in a discharge. The data at $t=817 \mathrm{~ms}$ correspond to a time before the electrode bias is applied, and the increase in plasma flow during bias at $t=819 \mathrm{~ms}$ is evidenced by the set of $I_{\text {sat }}$ points being drawn down and to the left.

$$
\begin{aligned}
\Gamma_{I H}(M, \theta)= & \Gamma_{0} \exp \left\{M \left[\left(1-\cos \left(\theta-\theta_{f}\right)\right) K_{u}\right.\right. \\
& \left.\left.-\left(1+\cos \left(\theta-\theta_{f}\right)\right) K_{d}\right]\right\} .
\end{aligned}
$$

The two constants in this expression are given by $K_{u}=0.64$ and $K_{d}=0.7$; these two numbers can be considered the calibration factors for this model. This expression is appropriate for $T_{i} / T_{e}$ ratios from 0.1 to 10 and flow speeds $M$ from 0 to 2.5.

In the analysis of data from HSX, the three parameters $\Gamma_{0}, M$, and $\theta_{f}$ are treated as fit parameters, which are used to fit Eq. (2) to the six $I_{\text {sat }}$ measurements at each time point using the Levenberg-Marquardt algorithm. ${ }^{30}$ This algorithm has the advantage of automatically providing uncertainty estimates for the fit parameters, provided the uncertainties in the signal are known. The uncertainty in each signal is taken to be the standard deviation of that individual $I_{\text {sat }}$ trace during a $5 \mathrm{~ms}$ window during the discharge when the electrode is not energized.

An example of these fits is shown in Fig. 3. The time slice at $817 \mathrm{~ms}$ refers to a time before the bias probe is energized; the plasma is only slowly flowing at this time. The time slice at $819 \mathrm{~ms}$ corresponds to well into the bias pulse, when the plasma is flowing more quickly. Note that the set of six $I_{\text {sat }}$ points is pulled farther to the bottom left at $819 \mathrm{~ms}$, when the bias is on, corresponding to greater plasma flow. The fit function represents the data well, both before and during the biased electrode pulse. The Mach numbers of the flows are measured to be 0.3 before the bias pulse is applied and 0.7 during the bias pulse. The uncertainties in the Mach number are also shown, based upon the uncertainties in the fit parameters from the curve fitting routine. Note that the Mach numbers are typically not converted to actual flow speeds, as the sound speed is not well known in HSX. 


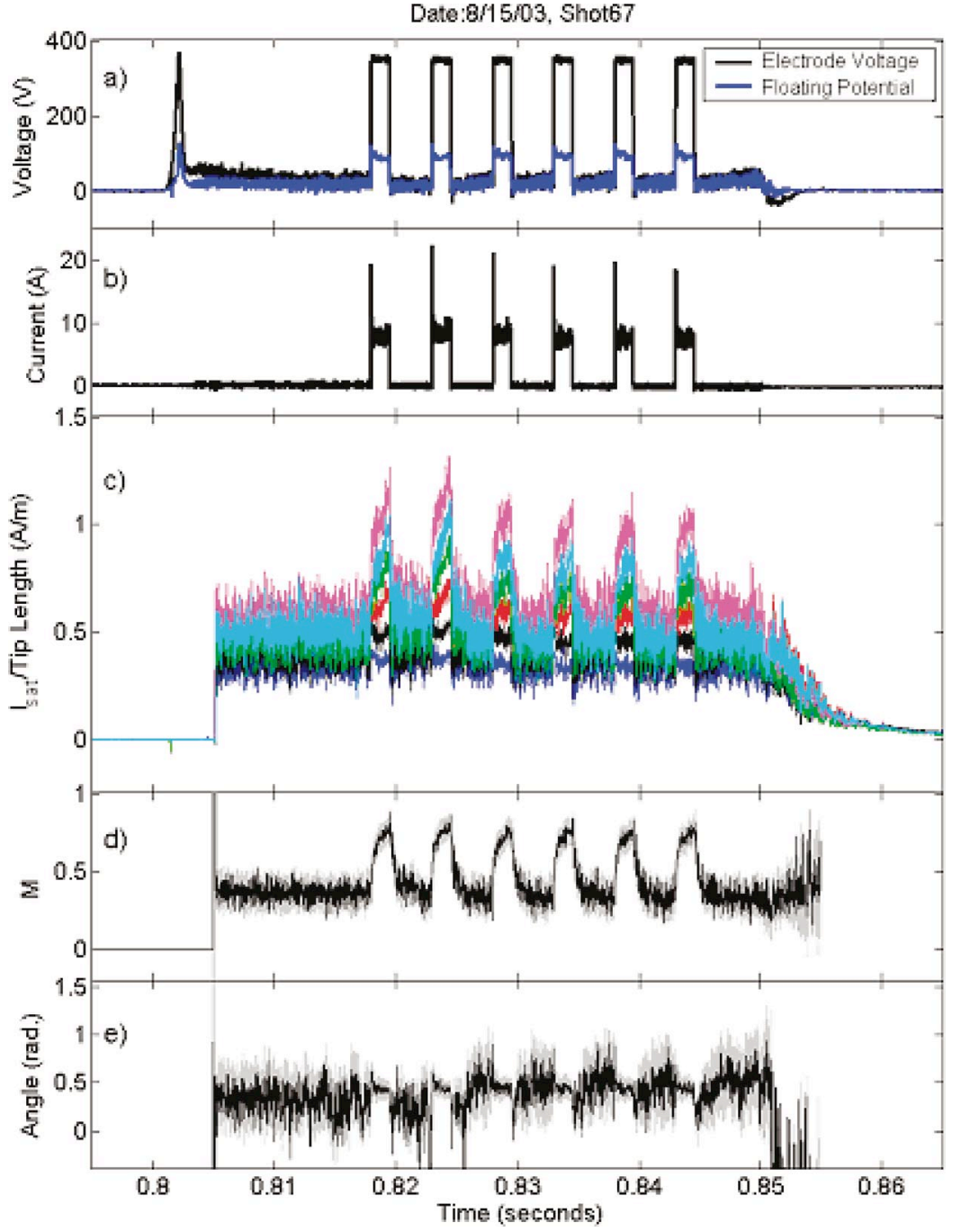

FIG. 4. (Color) Example of the evolution of the biasing and plasma parameters over an entire discharge. There are six bias pulses during the discharge, and the plasma responds similarly to each bias pulse. Signals shown are (a) electrode voltage and floating potential (b) electrode current, (c) the six $I_{\text {sat }}$ signals from the Mach probe, (d) the flow speed, and (e) the flow angle.

\section{EXAMPLE APPLICATION OF THE SYSTEM}

To present the utility of the system for these flow studies, data will be presented from discharges with a line averaged density of $1 \times 10^{12} \mathrm{~cm}^{-3}$ and $50 \mathrm{~kW}$ of ECH power. The biased electrode is fired six times during stationary conditions in each plasma discharge, with a pulse duration of 1.5 ms. The biased electrode is located at $r / a \approx 0.65$, while the low field side Mach probe is located at $r / a \approx 0.8$. All of the relevant signals from the probes and power supplies are shown in Fig. 4. A brief discussion of the signals in this figure is given now, with more detailed discussions of the individual parts following.

The top frame illustrates the voltage on the electrode and floating potential at the Mach probe. The floating potential evolution closely tracks the externally imposed electrode voltage, illustrating that the plasma is indeed biased by the electrode. The second frame illustrates the electrode current evolution. Note the large initial spike in the electrode current before it settles to the steady state value.

The third frame displays the evolution of the six $I_{\text {sat }}$ signals as measured by the low field side Mach probe. As described earlier, the separation in magnitude of the six signals illustrates that the plasma is flowing. The increasing separation during the electrode pulse is indicative of an increase in the flow speed. It has been observed that the $I_{\text {sat }}$ bias sometimes interferes with the breakdown of the plasma when the probes are deeply inserted. To avoid this problem, the $I_{\text {sat }}$ bias is applied via reed relays $5 \mathrm{~ms}$. after the ECH is turned on.

These six $I_{\text {sat }}$ signals translate into flow speed and direction waveforms as illustrated in the bottom two frames. These frames plot the fit parameters $M$ and $\theta_{F}$ as a function of time for this discharge. In each case, the uncertainty in the fit parameters is plotted as a gray envelope surrounding the trace. Note that the uncertainty envelope on both signals decreases during the bias pulse. This is because the locus of $I_{\text {sat }}$ points is pulled farther away from a circle, leading to a stronger constraint of the speed and angle. The flow angle in this figure is adjusted so that $0^{\circ}$ corresponds to the direction of the local magnetic field.

Detailed electrode voltage and current wave forms are displayed in Fig. 5. The signals from 18 separate realizations of the bias pulse (three reproducible discharges $\times$ six pulses per discharge) are averaged to provide these wave forms. Considering first the application of the electrode potential, the voltage on the electrode goes to its steady state of $350 \mathrm{~V}$ in approximately $1 \mu \mathrm{s}$. The electrode current shows a large spike of $\sim 19$ A before settling to a steady state value of 7.4 


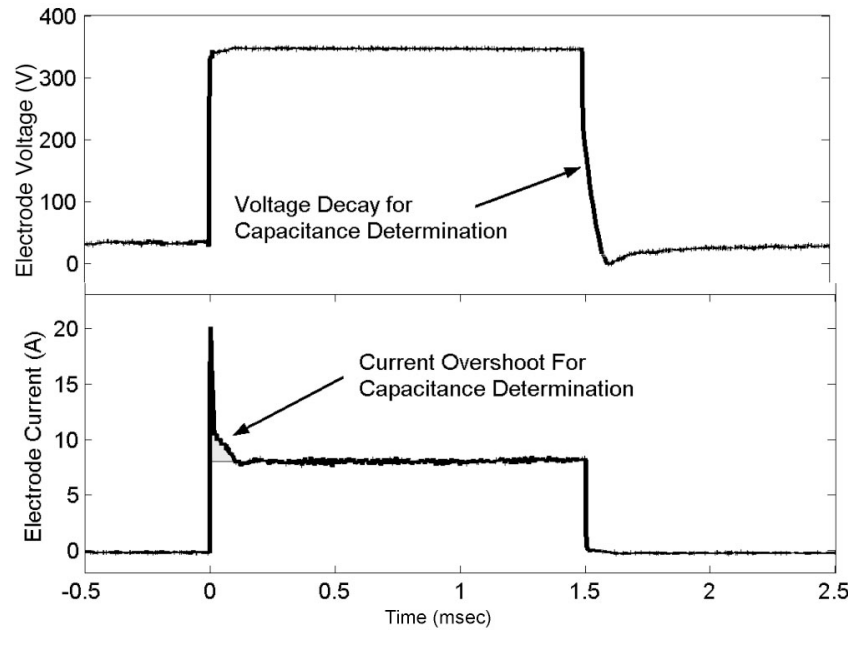

FIG. 5. Detailed evolution of (a) the electrode voltage and (b) the current during and after the bias pulse. Either the transient current at the beginning of the electrode pulse or the slow decay of the potential at the end of the bias allow for a determination of the effective capacitance of the biased plasma.

A. At bias turn off, the electrode current is terminated in $\sim 1 \mu \mathrm{s}$ by the opening of the IGBT switches. Once the switches are opened, the probe acts as a large floating potential monitor. The floating potential decays in $\sim 30 \mu$ s after bias termination, a time determined by the plasma parameters.

When a plasma contained in a magnetic field is biased by an electrode, the resulting biased state can be understood as a leaky capacitor with a plasma dielectric. ${ }^{6}$ In this model, the plates of the capacitor are the vessel wall and the magnetic surface upon which the biased electrode resides, and the leakage current is determined by the radial conductivity of the plasma. ${ }^{16}$ The electrode voltage and current wave forms allow the determination of the characteristics of this "capacitor." In the spin up phase, the area under the transient current (shaded in gray in the bottom frame of Fig. 5) repre- sents the total charge required to energize the capacitor. In the example shown, this represents $\sim 2.3 \times 10^{-4} \mathrm{C}$. This implies a capacitance for the system of $C=Q / V=6.5 \times 10^{-7} \mathrm{~F}$. The steady state "resistance" seen by the electrode is $R$ $\approx 46 \Omega$. When the current is terminated, the voltage decays with a time constant of $35 \mu \mathrm{s}$. This implies a capacitance of $C=35 \times 10^{-6} / 45 \approx 7.8 \times 10^{-7} \mathrm{~F}$. The capacitances calculated from the beginning and ending transients are thus very similar. Given the large aspect ratio of $\operatorname{HSX}(R / a \sim 10)$, the "vacuum capacitance" of the system can be approximated as a cylindrical capacitor. This calculation implies a plasma dielectric constant of $\sim 1000$. An estimation of the low frequency dielectric constant based on the plasma density and magnetic field strength $\left(\varepsilon_{\perp}=n m / \varepsilon_{0} B^{2}\right)$ yields a dielectric constant of $\sim 500$. This factor of 2 agreement seems reasonable, given the approximations made in the calculations.

Consider next the evolution of the flow as the biased electrode is energized. Each of the six $I_{\text {sat }}$ signals is averaged over the bias pulses to reduce the noise. The evolution of the locus of the six $I_{\text {sat }}$ values over the flow rise is shown in Fig. 6. The top left frame corresponds to the flow at the point when the electrode is energized. Subsequent times show the locus of points pull down and to the left as the flow accelerates. The trajectory of the series of points illustrates that the flow speed is increasing, but that the direction of flow is not changing significantly. The fit of Eq. (2) is shown at each time point, illustrating that the Hutchinson model fit works well at all points during the spin up process. The arrows represent the direction of the flow and their length is a relative measure of the flow speed, illustrating the evolution of the flow.

The time evolution of the flow speed and angle fit parameters is shown in Fig. 7. The flow Mach number is illustrated in the top frame, while the flow angle is illustrated in the bottom frame. The duration of the biased electrode pulse is denoted by the gray area. The vertical dashed lines corre-
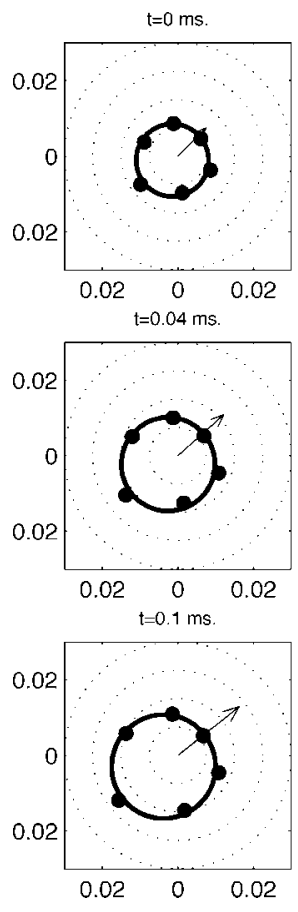
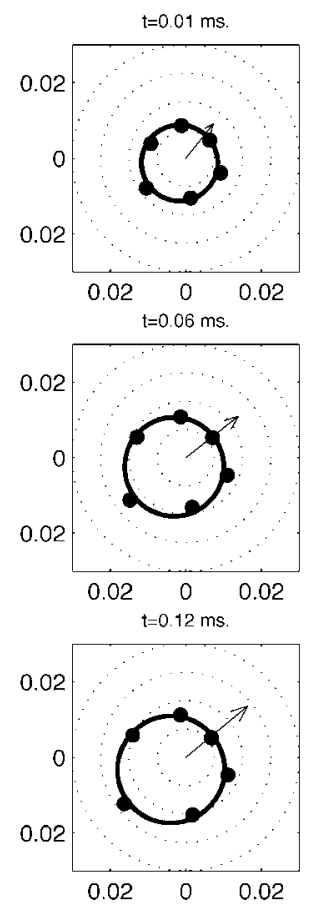
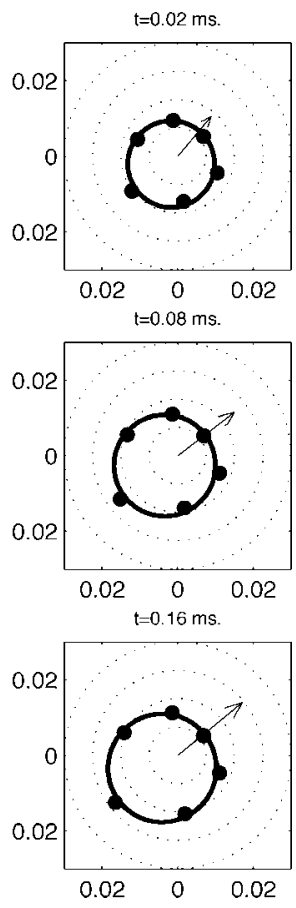

FIG. 6. Evolution of the locus of $I_{\text {sat }}$ values as the plasma spins-up. The points draw down and to the left, indicating the increasing plasma flow. The Hutchinson model fits are shown as a solid line, illustrating the quality of the fits throughout the spin-up process 

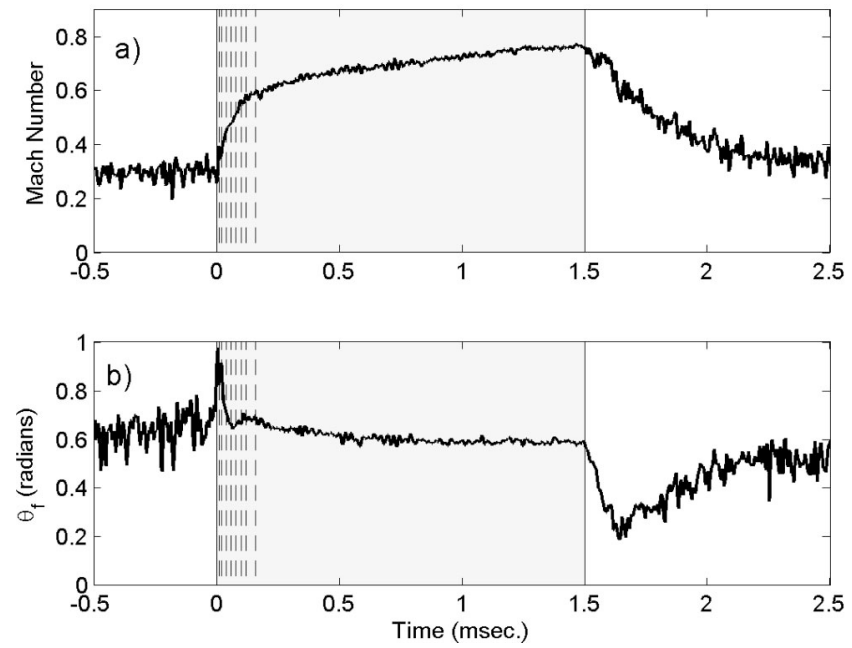

FIG. 7. Evolution of (a) the flow speed and (b) flow angle as the plasma spins up at bias turn-on and spins down at bias turn off. Note the large change in flow speed and the more subtle changes in flow angle as the plasma accelerates to its new steady state. The vertical dashed lines represent the times when the data in Fig. 6 was selected.

spond to the times illustrated in Fig. 6. There is a clear rise in the flow speed when the biased electrode voltage is applied, followed by a decay in the flow when the electrode current is terminated. The flow angle has a small excursion at bias turn on, and the flow angle during the bias pulse is rotated by $\sim 10^{\circ}$ compared to before the bias. As in Fig. 3, the flow angle in this figure is adjusted so that $0^{\circ}$ corresponds to the direction of the local magnetic field.

Simply speaking, the flow appears to rise with a time scale of $\sim 300 \mu \mathrm{s}$. A more detailed inspection of the flow speed and direction evolution reveals that there are two time scales present in the flow rise; a fast time scale of $\sim 20 \mu$ s or faster, and a longer time scale of $\sim 300 \mu \mathrm{s}$. A two time scale flow evolution in response to a biased electrode has been predicted based on neoclassical theory, ${ }^{31}$ and the rapid response of the probes allows detailed comparisons between neoclassical modeling and experiment. Data have also been collected in different configurations of HSX, allowing the study of how magnetic geometry impacts flow damping. These topics will be discussed in further publications.

It is important to verify that the measurements satisfy the radial force balance equation, given by

$$
\boldsymbol{E}_{r}+\boldsymbol{V} \times \boldsymbol{B}=\nabla p .
$$

During electrode bias, the ratio of the electric field term to the pressure gradient term can be estimated as

$$
\begin{aligned}
\frac{\nabla p}{E_{r}} \sim \frac{N_{i} T_{i}}{\Phi} \frac{L_{\Phi}}{L_{N}} & \sim \frac{1 \times 10^{18} 1.6 \times 10^{-19} 20}{300} \frac{.03}{0.06} \\
& \approx 5 \times 10^{-3} .
\end{aligned}
$$

$L_{\Phi}$ is potential scale length and is taken as the distance that the electrode is inserted inside the last closed surface $(0.03$ $\mathrm{m}) . L_{N}$ is the density scale length, and is approximately the system size $(0.06 \mathrm{~m}) . N_{i}$ is the plasma density (1 $\left.\times 10^{18} \mathrm{~m}^{-3}\right), \Phi$ is the plasma potential $(\sim 300 \mathrm{~V})$, and $T_{i}$ is the ion temperature. The ion temperature has been measured to be $\sim 20 \mathrm{eV}$ in HSX plasmas using impurity Doppler spec-

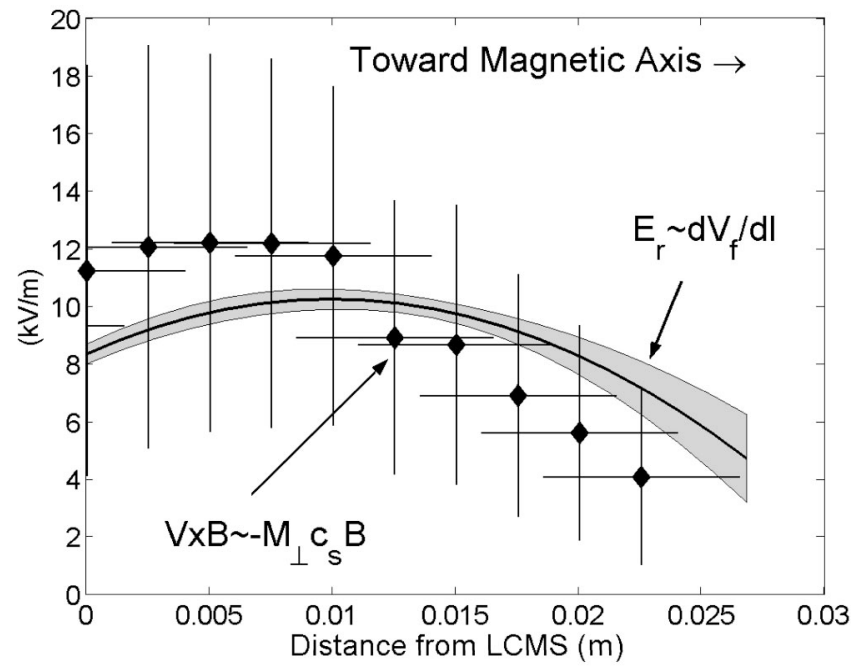

FIG. 8. Comparison of $\boldsymbol{E}_{r}$ and $-\boldsymbol{V} \times \boldsymbol{B}$ under $350 \mathrm{~V}$ of electrode bias at a line average density of $1 \times 10^{12} \mathrm{~cm}^{-3}$. The electric field is determined from the floating potential gradient and the $\boldsymbol{V} \times \boldsymbol{B}$ term from the Mach probe data. The large error bars in the $\boldsymbol{V} \times \boldsymbol{B}$ term are due to uncertainties in the electron temperature and the angle of the Mach probe with respect to the magnetic field.

troscopy, and there is no observation of a strong ion temperature gradient. This shows that the pressure gradient can be neglected under these circumstances.

The remaining terms in the expression can be evaluated based on data taken with the Mach probes. To estimate the electric field, we simply differentiate the floating potential profile as measured by scanning the probe on a shot to shot basis in similar discharges. The electron temperature gradient is observed to be small compared to the potential gradient induce by the large electrode, so this means of estimating the electric field should be reasonably accurate. The $\boldsymbol{V} \times \boldsymbol{B}$ term is estimated as the steady state Mach number of the perpendicular flows multiplied by the field strength $(B=0.5 \mathrm{~T})$ and sound speed as defined in the text before Eq. (2).

The comparison between the $\boldsymbol{E}_{r}$ and $\boldsymbol{V} \times \boldsymbol{B}$ terms is shown in Fig. 8. The agreement is observed to be quite reasonable, both in magnitude and profile shape. The rather large error bars in $\boldsymbol{V} \times \boldsymbol{B}$ are due to both the uncertainty in the electron temperature and the uncertainty in the angle alignment of the probe with the magnetic field in the highly shaped plasma.

A concern when using probes and electrode diagnostics is the perturbation to the plasma parameters due to these probes. This concern has been studied in HSX by measuring the flow features with either one or two Mach probes inserted into the plasma. The measured flow parameters (flow rates and time scales) are the same under both conditions. Hence, we infer than the presence of the Mach probes does not adversely affect the biased plasma flows.

Turbulence studies in the presence of electrode bias have also begun. HSX has not observed a biased $H$-mode in traditional sense, ${ }^{7}$ but a reduction in the amplitude of low frequency $(<100 \mathrm{kHz})$ floating potential fluctuations has been observed during the bias pulse. Further studies of the effect of the electrode bias on plasma turbulence will be performed in the future. 


\section{ACKNOWLEDGMENTS}

The authors would like to thank A. F. Almagri, W. Guttenfelder, K. M. Likin, and P. H. Probert for helpful discussion about the probe and electrode designs, the electronics, and the experiments conducted. Much gratitude is due to $\mathrm{M}$. Frankowski for providing essential help with the construction of the electrode and probes, A. Piccione for layout out the Mach probe electronics, and E. Jolitz for expertly operating HSX for the data presented in this article. This work was sponsored by the U.S. Department of Energy.

${ }^{1}$ F. S. B. Anderson, A. F. Almagri, D. T. Anderson, P. G. Mathews, J. N. Talmadge, and J. L. Shohet, Fusion Technol. 27, 273 (1995).

${ }^{2}$ A. H. Boozer, Phys. Plasmas 5, 1647 (1998).

${ }^{3}$ J. Nuhrenberg and R. Zille, Phys. Lett. A 129, 113 (1988).

${ }^{4}$ K. C. Shaing and J. D. Callen, Phys. Fluids 26, 3315 (1983).

${ }^{5}$ K. H. Burrell, Phys. Plasmas 4, 1499 (1997).

${ }^{6}$ J. G. Gorman and L. H. Th. Rietjens, Phys. Fluids 9, 2504 (1966).

${ }^{7}$ R. J. Taylor et al., Phys. Rev. Lett. 63, 2365 (1989).

${ }^{8}$ R. R. Weynants et al., Nucl. Fusion 32, 837 (1992).

${ }^{9}$ D. A. Diebold, N. Hershkowitz, J. Pew, J. Sorensen, T. Tanaka, R. Walsh,

E. Y. Wang, X. Wang, and G. Winz, Rev. Sci. Instrum. 66, 434 (1995).

${ }^{10}$ L. G. Askinazi, V. E. Golant, S. V. Lebedev, V. A. Rozhanskij, and M. Tendler, Nucl. Fusion 32, 271 (1992).

${ }^{11}$ G. S. Kirnev et al., Plasma Phys. Controlled Fusion 45, 337 (2003).

${ }^{12}$ S. Silva, H. Figueiredo, I. Nedzelsky, B. Goncalves, J. A. C. Cabral, C. A. F. Varandas, and G. Van Oost, Plasma Phys. Controlled Fusion 46, 163 (2004).
${ }^{13}$ L. Tramontin et al., Plasma Phys. Controlled Fusion 44, 195 (2002).

${ }^{14}$ A. F. Almagri, J. T. Chapman, C. S. Chiang, D. Craig, D. J. Den Hartog, C. C. Hegna, and S. C. Prager, Phys. Plasmas 5, 3982 (1998).

${ }^{15}$ E. Thomas, Jr., S. Knowlton, R. Gandy, J. Cooney, D. Pritchard, and T. Pruitt, Phys. Plasmas 5, 3991 (1998).

${ }^{16}$ J. N. Talmadge et al., Proceedings of the 15th International Conference on Plasma Physics and Controlled Fusion Research (Seville, 1994), IAEA, Vienna, 1995 Vol. 1, p. 797.

${ }^{17}$ A. Thorne, U. Litzen, and S. Johansson, Spectrophysics (Springer, Berlin, 1999), p. 191.

${ }^{18}$ R. P. Seraydarian, K. H. Burrell, N. H. Brooks, R. J. Groebner, and C. Kahn, Rev. Sci. Instrum. 57, 155 (1986).

${ }^{19}$ D. J. Den Hartog and R. J. Fonck, Rev. Sci. Instrum. 65, 3238 (1994).

${ }^{20}$ C. S. MacLatchy, C. Boucher, D. A. Poirier, and J. Gunn, Rev. Sci. Instrum. 63, 3923 (1992).

${ }^{21}$ B. J. Peterson, J. N. Talmadge, D. T. Anderson, F. S. B. Anderson, and J. L. Shohet, Rev. Sci. Instrum. 65, 2599 (1994).

${ }^{22}$ R. Back and R. D. Bengtson, Rev. Sci. Instrum. 68, 377 (1997).

${ }^{23}$ J. P. Gunn et al., Phys. Plasmas 8, 1995 (2001).

${ }^{24}$ K. S. Chung and I. H. Hutchinson, Phys. Rev. A 38, 4721 (1988), and references therein.

${ }^{25}$ M. Hudis and L. M. Lidsky, J. Appl. Phys. 41, 5011 (1970).

${ }^{26}$ I. H. Hutchinson, Phys. Plasmas 9, 1832 (2002).

${ }^{27}$ I. H. Hutchinson, Plasma Phys. Controlled Fusion 44, 1953 (2002).

${ }^{28}$ M. G. Shats, D. L. Rudakov, R. W. Boswell, and G. G. Borg, Phys. Plasmas 4, 3629 (1997).

${ }^{29}$ S. C. Hsu, T. A. Carter, G. Fiksel, H. Ji. R. M. Kulsrud, and M. Yamada, Phys. Plasmas 8, 1916 (2001).

${ }^{30}$ P. R. Bevington and D. K. Robinson, Data Reduction and Error Analysis for the Physical Sciences (McGraw-Hill, New York, 1992), p. 161.

${ }^{31}$ M. Coronado and J. N. Talmadge, Phys. Fluids B 5, 1200 (1993). 